\title{
Interpretation of Amniotic Fluid White Blood Cell Count in "Bloody Tap" Amniocenteses in Women With Symptoms of Preterm Labor
}

\author{
Sonya S. Abdel-Razeq, MD, Irina A. Buhimschi, MD, Mert O. Bahtiyar, MD, Victor A. \\ Rosenberg, MD, Antonette T. Dulay, MD, Christina S. Han, MD, Erika F. Werner, MD, \\ Stephen Thung, MD, and Catalin S. Buhimschi, MD \\ Department of Obstetrics, Gynecology and Reproductive Sciences, Yale University School of \\ Medicine, New Haven, Connecticut
}

\begin{abstract}
Objective-To estimate whether blood-contaminated amniotic fluid affects the performance of white blood cell (WBC) count in diagnosing intraamniotic inflammation and infection.

Methods-Three hundred fifty-seven consecutive women pregnant with singletons undergoing amniocentesis to rule out infection were enrolled prospectively. A "bloody tap" was defined as a red blood cell (RBC) count of 1,000 cells $/ \mathrm{mm}^{3}$ or more. Proteomics analysis of amniotic fluid was used in this study as the standard for diagnosing inflammation. Infection was confirmed by positive amniotic fluid culture. An amniotic fluid WBC count correction formula was computed using maternal WBC count, hematocrit, and mean corpuscular volume.
\end{abstract}

Results-The prevalence of a bloody tap amniocentesis was $22 \%$ (77 of 357). In the absence of inflammation, the amniotic fluid WBC count was significantly higher in bloody tap (median [interquartile range] 18 [9-58] cells $/ \mathrm{mm}^{3}$ ) compared with non-bloody tap specimens (4 [1-10] cells $\left./ \mathrm{mm}^{3} ; P<.001\right)$. The correction formula reversed this difference to a nonsignificant level (bloody tap 0 [0-17] compared with non-bloody tap $3[1-10]$ cells $/ \mathrm{mm}^{3} ; P=.273$ ). In the setting of inflammation, the observed WBC count of bloody tap samples $\left(778\left[197-2,062\right.\right.$ cells $\left.\left./ \mathrm{mm}^{3}\right]\right)$ was significantly elevated compared with that of the non-bloody tap specimens (616 [105-1,730] cells $\left./ \mathrm{mm}^{3} ; P=.023\right)$. Correction of the WBC count in bloody tap amniocenteses improved the test accuracy and positive likelihood ratios for inflammation and infection. A correction algorithm was not useful in amniotic fluid specimens with less than 1,000/RBCs $/ \mathrm{mm}^{3}$ or WBC counts more than 1,100 cells $/ \mathrm{mm}^{3}$. Given the nonlinear relationship between amniotic fluid WBC and RBC, for a rapid correction of WBC count, the number of neutrophils that need to be subtracted from the observed WBC count is variable.

(C) 2010 by The American College of Obstetricians and Gynecologists. Published by Lippincott Williams \& Wilkins.

Corresponding author: Sonya S. Abdel-Razeq, MD, Yale University, Department of Obstetrics, Gynecology \& Reproductive Sciences, 333 Cedar Street, LLCI 804, New Haven, CT 06520; sonya.abdel-razeq@yale.edu.

Financial Disclosure: The authors did not disclose any potential conflicts of interest. 
Conclusion-In the setting of an amniotic fluid sample contaminated with 1,000 RBCs $/ \mathrm{mm}^{3}$ or more, WBC count is a less accurate indicator of inflammation and infection. In such samples, correction of WBC count enhances diagnostic performance for inflammation and infection.

\section{Level of Evidence-II}

Compelling data suggest that in the setting of a maladaptive inflammatory ${ }^{1,2}$ response to bacterial invasion of the amniotic cavity, prolongation of pregnancy carries the risk of harmful effects for both the mother and fetus. ${ }^{3,4}$ When the clinical diagnosis of chorioamnionitis is obvious, the initiation of broad-spectrum antibiotic treatment and immediate delivery of the fetus is mandatory. ${ }^{4,5}$ However, maternal symptoms in intraamniotic infection are nonspecific and have a low accuracy in diagnosing histologic chorioamnionitis, a strong predictor of adverse neonatal outcome. ${ }^{6-9}$ Thus, suspicion of subclinical chorioamnionitis deems amniocentesis followed by direct analysis of amniotic fluid, which currently remains the most accurate method to assess for the presence or absence of intraamniotic infection. ${ }^{10-12}$

A positive bacterial culture is considered the gold standard for diagnosing intraamniotic infection. ${ }^{13,14}$ Unfortunately, culture results are not immediately available and proteomic analysis of the amniotic fluid, the most accurate diagnostic test for intraamniotic inflammation, is only available in research settings. ${ }^{15}$ Thus, clinicians often rely on amniotic fluid white blood cell (WBC) count as part of their criteria for a rapid workup of infectioninduced inflammation. Arguments in favor of amniotic fluid WBC count are its simple quantification using standard automated or manual cell counter techniques and its biologic relevance because the majority of amniotic fluid neutrophils are of fetal origin and thus closely reflect the fetal inflammatory response to infection. ${ }^{16}$ However, interpretation of WBC count in the setting of amniotic fluid contamination with blood ("bloody tap") is clinically difficult. ${ }^{17}$ We examined whether in vivo contamination of amniotic fluid with blood affects performance of WBC count in diagnosing intraamniotic inflammation and infection. Additionally, we investigated whether a correction formula has practical use in the setting of a bloody tap.

\section{Methods}

Our study population consisted of 357 consecutive women with singleton pregnancies, who presented to Yale-New Haven Hospital with symptoms of preterm labor, preterm premature rupture of membranes, or both from May 2004 to June 2009. All women underwent a clinically indicated amniocentesis for evaluation of intraamniotic infection and inflammation independent of our research protocol. The Human Investigational Committee of Yale University School of Medicine approved our study protocol and written informed consent was obtained from all participants before the procedure. Additional information regarding indications for amniocentesis procedures, exclusion criteria, and definitions of preterm labor and preterm premature rupture of membranes is provided in the Appendix available online at http://links.lww.com/AOG/A189.

After amniocentesis, an aliquot of amniotic fluid was immediately transported to the Yale hematology laboratory where it was examined in a Neubauer hemocytometer chamber for 
determination of the WBC and red blood cell (RBC) counts. Amniotic fluid was sent to the chemistry laboratory for assays of glucose concentration and L-lactate dehydrogenase activity. The Yale-New Haven Hospital microbiology laboratory performed the Gram stain and amniotic fluid cultures for aerobic and anaerobic bacteria as well as for Ureaplasma and Mycoplasma species, as previously described. ${ }^{13}$ The remaining amniotic fluid was transported to the research laboratory where it was centrifuged at $700 \mathrm{~g}$ for 10 minutes at $4^{\circ} \mathrm{C}$ and processed to confirm or exclude presence of intraamniotic inflammation with the use of mass spectrometry. ${ }^{15}$ The remaining amniotic fluid was frozen in aliquots at $-80^{\circ} \mathrm{C}$ for subsequent determination of the levels of the inflammatory cytokine interleukin- 6 using enzyme-linked immunosorbent assay) (Pierce Endogen, Rockford, IL).

A maternal venous blood specimen was collected by venipuncture at the time of amniocentesis and a complete blood cell count was performed by the same clinical laboratory, which reported amniotic fluid WBC and RBC counts. Previous studies indicate that maternal blood is responsible for the majority of the blood-contaminated amniocenteses specimens and that fetal blood accounts exclusively for only $4 \%$ of the cases. ${ }^{17,18}$ For the purpose of this study, the amniotic fluid WBC count reported by the Yale-New Haven Hospital clinical laboratory was considered the observed amniotic fluid WBC count. A predicted amniotic fluid WBC count was calculated on the basis of the maternal peripheral blood count and the amniotic fluid RBC count using the following formula: predicted amniotic fluid WBC count $\left(\right.$ cells $\left./ \mathrm{mm}^{3}\right)=$ amniotic fluid $\mathrm{RBC}$ count $\left(\right.$ cells $\left./ \mathrm{mm}^{3}\right) \times$ maternal blood WBC (cells $/ \mathrm{mm}^{3}$ )/maternal blood RBC count (cells $/ \mathrm{mm}^{3}$ ). The maternal RBC count was derived from the reported hematocrit and mean corpuscular volume using the following formula: maternal blood RBC count $\left(\right.$ cells $\left./ \mathrm{mm}^{3}\right)=$ hematocrit $(\%) \times 10^{7} /$ mean corpuscular volume (femtoliters). A corrected amniotic fluid WBC count was next calculated as the difference between the observed amniotic fluid WBC and the predicted amniotic fluid WBC count. Negative results were converted to 0 values and counted as overestimated instances. An observed-to-predicted ratio in amniotic fluid WBC counts was calculated as previously suggested for traumatic lumbar puncture specimens, with conversion of 0 results into values of $1 .{ }^{19}$

Presence of intraamniotic infection was established by a positive amniotic fluid culture result. In a previous prospective study, we demonstrated the high accuracy of proteomic analysis of the amniotic fluid to diagnose intraamniotic inflammation. ${ }^{20}$ Therefore, we used mass spectrometry surface-enhanced laser desorption ionization-time of flight to confirm or exclude intraamniotic inflammation, as previously described. ${ }^{15}$ The Mass Restricted score provides qualitative information regarding the presence or absence of inflammation and ranges from 0 to 4 , depending on the presence or absence of each of four protein biomarker peaks. ${ }^{15} \mathrm{~A}$ score of 3 or 4 indicates the presence of intraamniotic inflammation. All surfaceenhanced laser desorption ionization-time of flight assays were scored blindly by an investigator (I.A.B.) unaware of clinical presentation, laboratory results, or outcome.

As a result of the paucity of literature regarding criteria of what constitutes a bloody tap amniocentesis, we performed an experiment whereby peripheral blood samples from pregnant participants were diluted progressively to yield incremental concentrations of RBCs from 100 to 1 million cells $/ \mathrm{mm}^{3}$ as shown in Figure 1. We chose an amniotic fluid 
RBC count of 1,000 cells $/ \mathrm{mm}^{3}$ or greater to define a cytological bloody tap amniocentesis specimen, consistent with reports investigating blood-contaminated cerebrospinal fluid (ie, traumatic spinal tap). ${ }^{21,22}$

Our results were reported in accordance to the Standards for Reporting of Diagnostic Accuracy guidelines. ${ }^{23}$ Statistical analyses included one-way and two-way analysis of variance (ANOVA), Kruskal-Wallis ANOVA, $\chi^{2}$ analysis, McNemar's $\chi^{2}$, Spearman correlation, and receiver operating characteristic curve analysis, as previously described. ${ }^{24}$ Changes in diagnostic performance of amniotic fluid WBC count that could potentially result from application of the correction formula were evaluated using sensitivity, specificity, overall accuracy (ratio of cases correctly classified/total cases), graphic analysis of likelihood ratios, and matched sample agreement tables. ${ }^{25} \mathrm{~A} P$ value of $<.05$ was considered statistically significant. $P$ values were adjusted with the use of multiple logistic or linear regression analysis as appropriate. A detailed description of statistical methods, including analysis of likelihood ratios, is provided in the Appendix (http://

links.lww.com/AOG/A189).

\section{Results}

Of the 357 women enrolled in this study, 33\% (117 of 357) of the amniotic fluid samples met the definition for intraamniotic inflammation (Mass Restricted score 3-4). A flowchart of the enrolled patients and their clinical characteristics stratified by the bloody tap status presence or absence of intraamniotic inflammation is presented in the Appendix online at http://links.lww.com/AOG/A189.

Regarding the prevalence of samples with RBCs above the cutoffs resulting in visible discolorations of the samples, $11 \%$ (36 of 357) of the specimens had an RBC count greater than 6,000 cells $/ \mathrm{mm}^{3}$ (visually xanthochromic level) and $6 \%$ (21 of 357) had greater than 30,000 cells $/ \mathrm{mm}^{3}$ (visually bloody level, Fig. 1).

In Figure 2A, we represent the distribution of the observed amniotic fluid WBC count for the four study groups. Our analysis determined that in the absence of intraamniotic inflammation, the median amniotic fluid WBC count was significantly higher in bloody tap compared with non-bloody tap specimens (two-way ANOVA $P<.001$ ). Next, we analyzed the differences in observed WBC counts between the groups where inflammation was present and evaluated the number of amniotic fluid WBCs based on the bloody tap category. We established that in women with intraamniotic inflammation, contamination of amniotic fluid with blood resulted in a significant elevation of the observed WBC count compared with non-blood tap specimens $(P=.023)$. Given that we saw a significant interaction between intraamniotic inflammation and bloody tap status $(P=.046)$, we next sought to answer the question whether correction of amniotic fluid WBC count could be used to eliminate the possibility of bias in diagnosing intraamniotic inflammation (Fig. 2B). First, we found that after applying the correction formula, the previously noted differences in amniotic fluid WBC count registered in the absence of intraamniotic inflammation were rendered nonsignificant $(P=.273)$. The correction maintained, at the same significance level, the differences seen with inflammation per se (no inflammation compared with yes 
inflammation for both no and yes bloody tap specimens, $P<.001)$. Furthermore, our formula reversed the formerly observed significantly elevated amniotic fluid WBC of women with inflammation and bloody tap procedures compared with that of women with inflammation but non-bloody tap amniocenteses $(P=.701)$.

Numeric values for the corrected compared with observed amniotic fluid WBC counts for the four study groups are presented in Table 1. In a two-way repeated measure ANOVA, we determined that after applying the correction formula, WBC counts were lowered significantly in the groups with bloody tap procedures $(P<.001)$. Based on the results of microbial cultures, data analysis revealed similar differences in WBC counts observed both before (Fig. 2C) and after applying the correction algorithm (Fig. 2D). Numeric values for the observed amniotic fluid WBC and corrected amniotic fluid WBC counts stratified by the results of microbial cultures and bloody tap rank are also presented in Table 1.

Our next step was to analyze our data using the observed-to-predicted ratio of WBCs for the four categories of samples classified either by inflammation (Fig. 3A) or infection (Fig. 3B). We showed that in cases with intraamniotic inflammation, presence of a bloody tap resulted in a significant decrease in the observed-to-predicted WBC ratio compared with non-bloody tap specimens (Fig. 3A, $P<.001$ ). Furthermore, our analysis determined that the observed-topredicted WBC ratio is affected synergistically by both bloody tap and inflammatory status of the sample (two-way ANOVA, $P<.001$ ).

Using receiver operating characteristic analysis, we determined that in a non-bloody tap specimen, the cutoff with the highest accuracy in diagnosing inflammation was 31 WBCs $/ \mathrm{mm}^{3}$ (sensitivity [95\% confidence interval (CI)] 88\% [80-95], specificity 93\% [8996], positive likelihood ratio 11.7 [7.1-19.1], negative likelihood ratio 0.1 [0.1-0.2], accuracy 91 [88-94]). The cutoff with the highest accuracy to diagnose inflammation in a bloody tap specimen was $123 \mathrm{WBC} / \mathrm{mm}^{3}$ (sensitivity $84 \%$ [72-96], specificity 88\% [7798], positive likelihood ratio 6.7 [2.9-15.4], negative likelihood ratio 0.2 [0.1-0.4], accuracy 86 [78-94]). After applying the correction formula, the optimal WBC cutoff for the bloody tap specimens was lowered from 123 to $50 \mathrm{WBCs} / \mathrm{mm}^{3}$ (sensitivity 78\% [95-92], specificity 93\% [84-100], positive likelihood ratio 10.5 [3.5-31.4], negative likelihood ratio 0.2 [0.10.4 ], accuracy 86[78-94]). We determined that there was no statistical difference in areas under the three receiver operating characteristic curves for the three cutoffs described (observed WBC in no bloody tap compared with bloody tap: $\mathrm{z}$ statistic=0.493, $P=.622$; compared with corrected WBC: $\mathrm{z}$ statistic $=0.075, P=.123)$.

The optimal cutoff for the observed WBC count to diagnose infection in a bloody tap compared with a non-bloody tap specimen was slightly different (bloody tap: 22 WBCs $/ \mathrm{mm}^{3}$; non-bloody tap: $28 \mathrm{WBCs} / \mathrm{mm}^{3}$ ). The test performance of the observed WBC count to diagnose infection in a bloody tap sample was as follows: sensitivity $90 \%$ (95\% CI 80-100), specificity 46\% (95\% CI 31-60), positive likelihood ratio 1.7 (95\% CI 1.2-2.2), negative likelihood ratio 0.2 (95\% CI $0.1-0.7$ ), accuracy $64 \%$ (95\% CI 53-44). The test performance of the observed WBC count to diagnose infection in a non-bloody tap sample was: sensitivity 83\% (95\% CI 74-92), specificity 86\% (95\% CI 81-90), positive likelihood ratio 5.8 (95\% CI 4.1-8.1), negative likelihood ratio 0.2 (95\% CI $0.1-0.3$ ), accuracy $85 \%$ 
(95\% CI 81-89). Our analysis showed a significant decrease in area under the receiver operating characteristic curve for the observed WBC count in a bloody compared with a non-bloody tap specimen (z statistic $=0.187, P=.007$ ). This suggested a lower diagnostic performance of the WBC count in the setting of a bloody tap amniocentesis. Application of the correction formula with its own optimal cutoff $\left(30 \mathrm{WBCs} / \mathrm{mm}^{3}\right)$ improved the overall diagnostic performances and accuracy of the WBC count to diagnose infection (sensitivity $80 \%$ [95\% CI 68-930], specificity 64\% [95\% CI 48-80], positive likelihood ratio 2.2 [95\% CI 1.4-3.5], negative likelihood ratio 0.3 [95\% CI 0.2-0.6], accuracy 73\% [95\% CI 63-83]). Our correction raised the area under the receiver operating characteristic curve for the corrected WBC count to values not different from that of the observed WBC count in a nonbloody tap specimen (z statistic $=0.164, P=.101)$.

The test performances are reported in Table 2. First we found that the sensitivity of the observed WBC count was the highest in diagnosing intraamniotic inflammation and infection in bloody tap specimens compared with non-bloody tap cases. However, this was accompanied by a marked decrease in specificity. Correction of WBC count in the bloody tap population improved the specificity of the test. We next analyzed the overall accuracy of the three tests. We determined that the observed WBC had the best test accuracy to diagnose inflammation and infection in non-bloody tap compared with bloody tap specimens. This suggests that correction of the WBC count in samples with less than $1,000 \mathrm{RBC} / \mathrm{mm}^{3}$ is not necessary. However, compared with the observed WBC count, correction of the WBC count in bloody tap amniocenteses improved the overall test accuracy for both inflammation and infection. This suggests that correction of the WBC count in samples with $1,000 \mathrm{RBC} / \mathrm{mm}^{3}$ or greater is needed for a better diagnosis of inflammation/infection.

To substantiate our observations analytically and to calculate the levels of significance for the observed differences in sensitivity and specificity, we restricted our analysis to the bloody tap specimens by using the method of matched sample agreement tables. ${ }^{25}$

The ability of our corrective formula to improve the diagnostic performance of the WBC count in predicting intraamniotic infection in bloody tap specimens is presented next. Following calculation of the McNemar's $\chi^{2}$ values, we established that our method improved the sensitivity for all tested WBC cutoffs $\left(10 \mathrm{WBCs} / \mathrm{mm}^{3}: \chi^{2} 15.059, P<.001 ; 30\right.$ $\mathrm{WBCs} / \mathrm{mm}^{3}: \chi^{2} 9.091, P=.003 ; 50 \mathrm{WBCs} / \mathrm{mm}^{3}: \chi^{2} 8.100, P=.004 ; 100 \mathrm{WBCs} / \mathrm{mm}^{3}: \chi^{2}$ $4.167, P=.041)$. The correction neither improved test specificity for intraamniotic inflammation or infection nor modified the diagnostic performance of the observed WBC count in non- bloody tap specimens.

We subsequently performed a comparative analysis of the likelihood ratios calculated for the WBC count of bloody and non-bloody tap samples. A graphic representation of the results is displayed in Figure 4. Our analysis showed that there was no overlap between the 95\% CIs of the positive likelihood ratios (ascending lines) of the non-bloody tap (drawn in black) and bloody tap (drawn in red) specimens for the 10 (Fig. 4A), 30 (Fig. 4B), and 50 WBCs $/ \mathrm{mm}^{3}$ (Fig. 4C) cutoffs. This finding indicated a statistically significant difference at a level of at least $P<.05$. The correction method used in this study leads to a significant shift to the left of the positive likelihood ratio of the corrected WBC count (displayed in blue). This 
signified that the diagnostic performance of the corrected WBC count was improved. The size of the red vector, which is illustrative of the level of bias in diagnosing inflammation imposed by the bloody tap decreased with the elevation in the WBC threshold. The size of the blue vector, which is representative of the difference from the best possible clinical scenario (non-bloody tap procedure), decreased in a similar fashion. The overlap between the CIs, together with the small size of the red and blue vectors reflected a nonsignificant level of change in the likelihood ratios of the corrected WBC compared with observed WBC count when a cutoff 100 cells $/ \mathrm{mm}^{3}$ or greater was considered as indicative of inflammation (Fig. 4D).

In addition to the use of the arithmetic correction based on maternal hematological parameters (WBC count, hematocrit, and mean corpuscular volume), we sought to provide a simpler and faster solution for clinicians interested in diagnosing amniotic fluid inflammation and infection as accurately as possible. To do so, we first analyzed the relationship between the amniotic fluid RBC and WBC count. We determined that this relationship is nonlinear and best fits a log-log plot (Fig. 5A). This examination confirmed that a correction of the observed WBC count is not necessary in amniotic fluid samples contaminated with less than $1,000 \mathrm{RBCs} / \mathrm{mm} .^{3}$ Yet, blood contamination above this threshold requires different levels of WBC correction, which cannot be estimated by a simple $\mathrm{WBC} / \mathrm{RBC}$ ratio as a result of the nonlinear relationship between the two variables. Based on our calculations, in Figure 5B, we report the median number of WBCs that needs to be subtracted from the observed WBC count for a rapid correction of amniotic fluid leukocytosis in a woman with a bloody tap amniocentesis. We were next interested in determining the level of agreement between the corrected WBC counts calculated using the mathematical formula and the rapid method based on the WBC thresholds listed in Figure 5B. The kappa (95\% CI) coefficients of agreement for correct classification of samples (yes compared with no inflammation) based on the cutoffs of $10,30,50$, or $100 \mathrm{WBCs} / \mathrm{mm}^{3}$ were all classified in the "very good" range and are as follows: 0.87 (0.75-0.98), 0.87 (0.76$0.98), 0.95$ (0.87-1.00), and 0.95 (0.87-1.00), respectively. This rapid method of correction resulted in misclassification of five samples when cutoffs of 10 or $30 \mathrm{WBCs} / \mathrm{mm}^{3}$ were used to define inflammation. Only two instances remained misclassified when the 50 or 100 $\mathrm{WBCs} / \mathrm{mm}^{3}$ cutoffs were used. Specific examples accompanied by numeric calculations are provided in Box 1.

\section{Discussion}

Our research provides evidence that contamination of amniotic fluid with RBC more than 1,000 cells $/ \mathrm{mm}^{3}$ modifies the absolute WBC count in samples with and without proteomic biomarkers characteristic of inflammation. Our results suggest that by using a WBC count corrective formula in the setting of a bloody tap amniocentesis, the diagnostic uncertainty for inflammation and infection can be decreased. Mass spectrometry is not routinely available as a diagnostic tool in most clinical and research arenas. Thus, this algorithm may be especially useful in clinical scenarios in which a misleading elevated WBC count, arising from the procedure, leads to a false-positive diagnosis of intraamniotic inflammation.

Because clinical intervention based on a false-positive test result may be associated with significant potential maternal and neonatal morbidity, any means of decreasing the rate of 
bias for intraamniotic inflammation/infection should be undertaken. Additionally, our examination offers potential improvement in the quality of amniotic fluid research. That is, the prevalence of intraamniotic inflammation in the general population will be more accurately reported if bloody amniotic fluid samples are assessed and not deliberately excluded from data analysis.

Our correction method lowered WBC count in bloody tap samples, which had proven inflammation. However, in this particular group of samples, application of our corrective method does not affect the clinician's ability to correctly establish the presence of inflammation. This conclusion is supported by comparative analysis of the observed WBC compared with corrected WBC count in bloody tap samples with Mass Restricted scores 34. After correction, amniotic fluid WBC count remained above threshold values required to diagnose inflammation and infection. ${ }^{22,26-29}$ In our analysis, amniotic fluid WBC count greater than 1,100 cells $/ \mathrm{mm}^{3}$ reflected true amniotic fluid leukocytosis for which a correction was unnecessary. These results are consistent with those from neurology research showing true leukocytosis was rarely masked in blood contaminated cerebrospinal fluid when the adjusted neutrophil count was at least 10 times over the number of WBCs. ${ }^{30}$

The data derived from our analysis are novel in that we provide a comprehensive assessment of the value of WBC count correction in bloody amniotic fluid samples by using a variety of statistical tests and methods. We concluded that the ratio of the observed-to-predicted WBC count overcorrected amniotic fluid neutrophil count in both negative and positive inflammatory samples. Hence, adjustment of amniotic fluid WBC count using this ratio may increase the risk of a false-negative result and, thus, it may be of limited clinical usefulness.

Using receiver operating characteristic analysis, we established that the best WBC cutoff needed to diagnose inflammation and infection was dependent on the bloody status of the amniotic fluid. The first conclusion of this analysis was that if RBC count is estimated at less than 1,000 RBCs $/ \mathrm{mm}^{3}$, a correction becomes unnecessary and a cutoff of 31 WBCs $/ \mathrm{mm}^{3}$ could be used as optimal. For specimens with $1,000 \mathrm{RBCs} / \mathrm{mm}^{3}$ or greater, a higher WBC threshold was required $\left(123 \mathrm{WBCs} / \mathrm{mm}^{3}\right)$ for an optimal diagnosis. This cutoff was significantly higher compared with that required to diagnose inflammation in a nonbloody tap specimen. After implementation of a corrective formula, we demonstrated the best diagnostic threshold to diagnose inflammation in bloody samples is $50 \mathrm{WBCs} / \mathrm{mm}^{3}$ with no significant difference among areas under the three receiver operating characteristic curves for the three cutoffs described.

The desire to correctly distinguish negative, traumatic, and positive inflammatory amniotic fluid samples raises an important question: is the diagnosis of inflammation or infection better served based on mathematical formula compared with the use of higher WBC diagnostic threshold in samples contaminated with $1,000 \mathrm{RBC} / \mathrm{mm}^{3}$ or greater? We sense that implementation of a corrective mathematical calculation adds increased clinical difficulty and establishment of a diagnosis based on a higher cutoff may prove easier for clinicians. However, our results are consistent with similar areas under the curve for the observed WBC compared with corrected WBC cutoffs and are indicative of the strength of our analytic approach. 
Because a large variety of diagnostic tests is available, a comparative analysis of their diagnostic performance is needed. A traditional comparison considers sensitivity, specificity, and accuracy. ${ }^{25}$ The sensitivity of the observed WBC count was the highest in diagnosing intraamniotic inflammation and infection in bloody tap specimens compared with non-bloody tap cases. This observation was not a surprise given an elevation in the WBC count in bloody amniotic fluid specimens increased the proportion of cases correctly identified by the test. However, this sensitivity was at the expense of a marked decrease in both specificity and overall accuracy of the test. Conversely, correction of the WBC count in the bloody tap population significantly improved the overall accuracy of the WBC to correctly diagnose both inflammation and infection. This observation suggests that correction of WBC count in bloody amniotic fluid specimens may be clinically useful.

Unlike sensitivity and specificity, likelihood ratios are independent of the prevalence of the disease and have been increasingly preferred to sensitivity and specificity as measures of diagnostic performance. ${ }^{31}$ There is recent agreement that likelihood ratios have increased clinical relevance and are more practical in making sense of diagnostic test results. ${ }^{32} \mathrm{~A}$ likelihood ratio of 1.0 indicates no change from pretest probability, suggesting a useless test or test modification. A good positive likelihood ratio is more than 2, corresponding to the probability of disease of approximately $67 \% .{ }^{33}$ In the current study, we conclude that the WBC count is affected in bloody tap specimens to the level of a poorly performing test for both inflammation and infection as illustrated by positive likelihood ratios less than 2 . The benefit of the correction algorithm is demonstrated by a consistent improvement in the positive likelihood ratios for the corrected WBC in bloody tap specimens.

This analysis suggests that a correction algorithm is not worthwhile in amniotic fluid specimens with less than $1,000 / \mathrm{RBCs} \mathrm{mm}^{3}$ or WBC counts more than 1,100 cells $/ \mathrm{mm}^{3}$. To provide a simpler and faster solution for clinicians interested in rapid interpretation of amniotic fluid results for inflammation or infection, we analyzed the relationship between the degree of blood contamination and predicted WBC count in our population. We calculated the number of WBCs needed to be subtracted from the observed WBC count for a rapid correction of the number of amniotic fluid neutrophils. This provides an estimated corrected WBC count and can be used in conjunction with several WBC cutoffs for better diagnosis of inflammation and infection in women with bloody tap amniocenteses.

In summary, our data provide evidence that contamination of amniotic fluid with blood affects diagnostic performance of WBC count to confirm intraamniotic inflammation and infection. In amniotic fluid samples contaminated with $1,000 \mathrm{RBC} / \mathrm{mm}^{3}$ or greater, the proposed correction may help with removing false-positive bias among women without intraamniotic inflammation and infection. Nevertheless, the WBC count should be interpreted together with results of other rapid amniotic fluid tests. All available laboratory information should be evaluated carefully before a final management decision is reached and WBC count alone should not be a substitution for clinical judgment. 


\section{Acknowledgments}

Supported by the National Institutes of Health/Eunice Kennedy Shriver National Institute of Child Health and Human Development (NIH/NICHD) Grant RO1 HD 047321 (I. A. B.) and departmental funds. Dr. Buhimschi was supported by RO3 HD 50249 and the Yale WRHR Career Development Center (K12 HD 1027766) (CJL).

The authors thank the nurses, fellows, residents, and faculty at Yale-New Haven Hospital, the Department of Obstetrics and Gynecology and Reproductive Sciences, and all patients who participated in the study.

\section{References}

1. Buhimschi CS, Rosenberg VA, Dulay AT, Thung S, Sfakianaki AK, Bahtiyar MO, et al. Multidimensional system biology: genetic markers and proteomic biomarkers of adverse pregnancy outcome in preterm birth. Am J Perinatol. 2008; 25:175-87. [PubMed: 18425720]

2. Wu YW, Colford JM Jr. Chorioamnionitis as a risk factor for cerebral palsy: a meta-analysis. JAMA. 2000; 284:1417-24. [PubMed: 10989405]

3. Buhimschi CS, Dulay AT, Abdel-Razeq S, Zhao G, Lee S, Hodgson EJ, et al. Fetal inflammatory response in women with proteomic biomarkers characteristic of intraamniotic inflammation and preterm birth. BJOG. 2009; 116:257-67. [PubMed: 18947340]

4. Premature rupture of membranes. Clinical management guidelines for obstetrician-gynecologists. ACOG Practice Bulletin No. 80. American College of Obstetricians and Gynecologists. Obstet Gynecol. 2007; 109:1007-19. [PubMed: 17400872]

5. Gibbs RS, Duff P. Progress in pathogenesis and management of clinical intraamniotic infection. Am J Obstet Gynecol. 1991; 164:1317-26. [PubMed: 2035575]

6. Guzick DS, Winn K. The association of chorioamnionitis with preterm delivery. Obstet Gynecol. 1985; 65:11-6. [PubMed: 3966012]

7. Miyazaki K, Furuhashi M, Matsuo K, Minami K, Yoshida K, Kuno N, et al. Impact of subclinical chorioamnionitis on maternal and neonatal outcomes. Acta Obstet Gynecol Scand. 2007; 86:191-7. [PubMed: 17364282]

8. Bracci R, Buonocore G. Chorioamnionitis: a risk factor for fetal and neonatal morbidity. Biol Neonate. 2003; 83:85-96. [PubMed: 12576751]

9. Edmondson N, Bocking A, Machin G, Rizek R, Watson C, Keating S. The prevalence of chronic deciduitis in cases of preterm labor without clinical chorioamnionitis. Pediatr Dev Pathol. 2009; 12:16-21. [PubMed: 18171100]

10. Dudley J, Malcolm G, Ellwood D. Amniocentesis in the management of preterm premature rupture of the membranes. Aust N Z J Obstet Gynaecol. 1991; 31:331-6. [PubMed: 1799346]

11. Garite TJ, Freeman RK, Linzey EM, Braly P. The use of amniocentesis in patients with premature rupture of membranes. Obstet Gynecol. 1979; 54:226-30. [PubMed: 460758]

12. Broekhuizen FF, Gilman M, Hamilton PR. Amniocentesis for gram stain and culture in preterm premature rupture of the membranes. Obstet Gynecol. 1985; 66:316-21. [PubMed: 2410839]

13. Han YW, Shen T, Chung P, Buhimschi IA, Buhimschi CS. Uncultivated bacteria as etiologic agents of intra-amniotic intraamniotic inflammation leading to preterm birth. J Clin Microbiol. 2009; 47:38-47. [PubMed: 18971361]

14. Sperling RS, Newton E, Gibbs RS. Intraamniotic infection in low-birth-weight infants. J Infect Dis. 1988; 157:113-7. [PubMed: 3335795]

15. Buhimschi IA, Christner R, Buhimschi CS. Proteomic biomarker analysis of amniotic fluid for identification of intra-amniotic inflammation. BJOG. 2005; 112:173-81. [PubMed: 15663581]

16. Sampson JE, Theve RP, Blatman RN, Shipp TD, Bianchi DW, Ward BE, et al. Fetal origin of amniotic fluid polymorphonuclear leukocytes. Am J Obstet Gynecol. 1997; 176:77-81. [PubMed: 9024093]

17. Carlan SJ, Gearity DE, O'Brien WF, Roth L. The effect of maternal blood contamination on amniotic fluid glucose and white blood cell count from preterm gestations. Obstet Gynecol. 1996; 87:185-7. [PubMed: 8559520] 
18. Nuss S, Brebaum D, Grond-Ginsbach C. Maternal cell contamination in amniotic fluid samples as a consequence of the sampling technique. Hum Genet. 1994; 93:121-4. [PubMed: 8112733]

19. Mazor SS, McNulty JE, Roosevelt GE. Interpretation of traumatic lumbar punctures: who can go home? Pediatrics. 2003; 111:525-8. [PubMed: 12612231]

20. Buhimschi CS, Bhandari V, Hamar BD, Bahtiyar MO, Zhao G, Sfakianaki AK, et al. Proteomic profiling of the amniotic fluid to detect inflammation, infection, and neonatal sepsis. PLoS Med. 2007; 4:e18. [PubMed: 17227133]

21. Eskey CJ, Ogilvy CS. Fluoroscopy-guided lumbar puncture: decreased frequency of traumatic tap and implications for the assessment of CT-negative acute subarachnoid hemorrhage. AJNR Am J Neuroradiol. 2001; 22:571-6. [PubMed: 11237986]

22. Shah KH, Richard KM, Nicholas S, Edlow JA. Incidence of traumatic lumbar puncture. Acad Emerg Med. 2003; 10:151-4. [PubMed: 12574013]

23. Bossuyt PM, Reitsma JB, Bruns DE, Gatsonis CA, Glasziou PP, Irwig LM, et al. Standards for Reporting of Diagnostic Accuracy. Towards complete and accurate reporting of studies of diagnostic accuracy: the STARD initiative. BMJ. 2003; 326:41-4. [PubMed: 12511463]

24. Park SH, Goo JM, Jo CH. Receiver operating characteristic (ROC) curve: practical review for radiologists. Korean J Radiol. 2004; 5:11-8. [PubMed: 15064554]

25. Hawass NE. Comparing the sensitivities and specificities of two diagnostic procedures performed on the same group of patients. Br J Radiol. 1997; 70:360-6. [PubMed: 9166071]

26. Garry D, Figueroa R, Aguero-Rosenfeld M, Martinez E, Visintainer P, Tejani N. A comparison of rapid amniotic fluid markers in the prediction of microbial invasion of the uterine cavity and preterm delivery. Am J Obstet Gynecol. 1996; 175:1336-41. [PubMed: 8942511]

27. Edwards RK, Clark P, Locksmith Gregory J, Duff P. Performance characteristics of putative tests for subclinical chorioamnionitis. Infect Dis Obstet Gynecol. 2001; 9:209-14. [PubMed: 11916177]

28. Buhimschi CS, Sfakianaki AK, Hamar BG, Pettker CM, Bahtiyar MO, Funai E, et al. A low vaginal 'pool' amniotic fluid glucose measurement is a predictive but not a sensitive marker for infection in women with preterm premature rupture of membranes. Am J Obstet Gynecol. 2006; 194:309-16. [PubMed: 16458622]

29. Larsen JW, Goldkrand JW, Hanson TM, Miller CR. Intrauterine infection on an obstetric service. Obstet Gynecol. 1974; 43:838-43. [PubMed: 4829278]

30. Mayefsky JH, Roghmann KJ. Determination of leukocytosis in traumatic spinal tap specimens. Am J Med. 1987; 82:1175-81. [PubMed: 3605134]

31. Biggerstaff BJ. Comparing diagnostic tests: a simple graphic using likelihood ratios. Stat Med. 2000; 19:649-63. [PubMed: 10700737]

32. Attia J. Moving beyond sensitivity and specificity: using likelihood ratios to help interpret diagnostic tests. Aust Prescr. 2003; 26:111-3.

33. Wang ST, Pizzolato S, Demshar HP. Receiver operating characteristic plots to evaluate Guthrie, Wallac, and Isolab phenylalanine kit performance for newborn phenylketonuria screening. Clin Chem. 1997; 43:1838-42. [PubMed: 9342001] 


\section{Specific Examples Accompanied by Numeric Calculations of the White Blood Cell Correction Formulas for a Patient With Positive (Case 1) or Negative Intraamniotic Inflammation and Infection (Case 2)}

\section{Case 1 (positive inflammation and infection)}

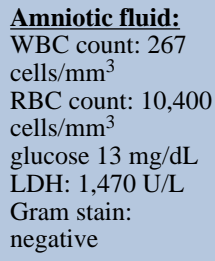

RBC count: 10,400

cells $/ \mathrm{mm}^{3}$

glucose $13 \mathrm{mg} / \mathrm{dL}$

LDH: $1,470 \mathrm{U} / \mathrm{L}$

Gram stain:

negative

\section{Maternal}

hematology:

WBC count: 14,200

cells $/ \mathrm{mm}^{3}$

Hematocrit: $28.9 \%$

MCV: $101 \mathrm{fL}$

MR score: 4
Predicted $W B C$ count $=10,400 \times \frac{14,200 \times 101}{28.9 \times 10^{7}}=52$ cells $/ \mathrm{mm}^{3}$

Corrected WBC count $=267-52=215$ cells $/ \mathrm{mm}^{3}$

Corrected WBC count (rapid subtraction $)=267-50=217$ cells $/ \mathrm{mm}^{3}$

Amniotic fluid culture: positive for Ureaplasma Urealyticum (final results reported 5 days after amniocentesis)

\section{Case 2 (negative inflammation and infection)}

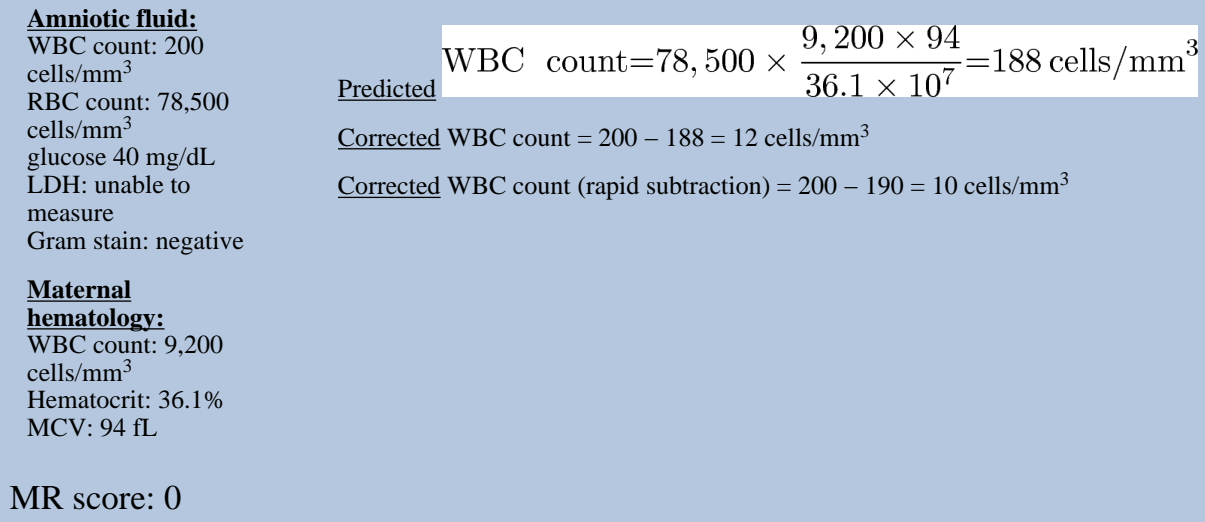

MR score: 0

Amniotic fluid culture: negative (final results reported 7 days after amniocentesis)

WBC, white blood cells; RBC, red blood cells; LDH, lactate dehydrogenase activity; MCV, mean corpuscular volume; fL, femtoliters; MR score, Mass Restricted proteomics score. 


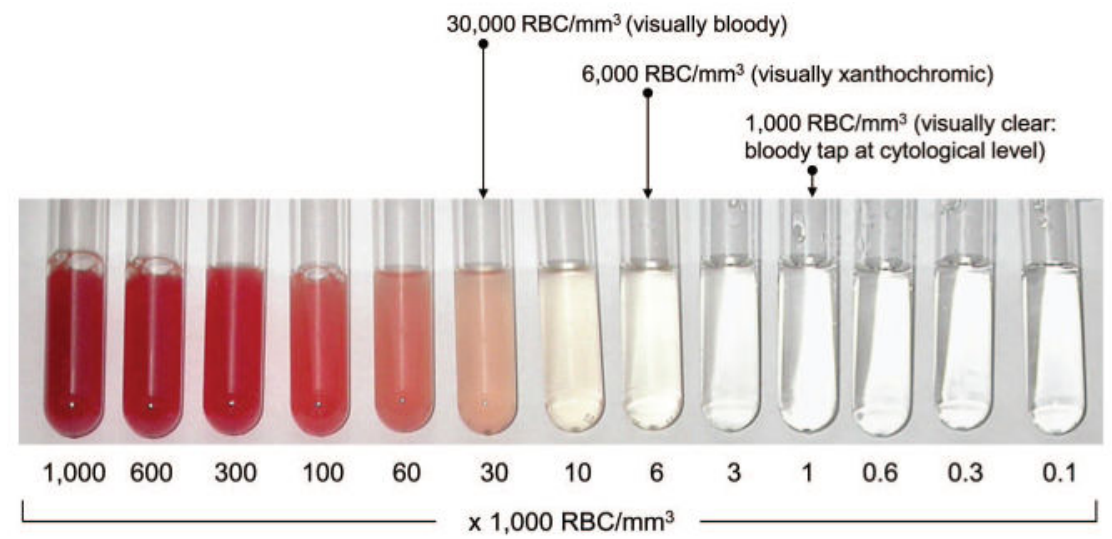

Fig. 1.

Experiment performed to distinguish cytologic compared with macroscopic blood contamination of the amniotic fluid at a subjective level of xanthochromic $(6,000$ red blood cells $\left.\left.[\mathrm{RBCs}] / \mathrm{mm}^{3}\right]\right)$ and frank $\left(30,000 \mathrm{RBCs} / \mathrm{mm}^{3}\right)$ bloody appearance. 


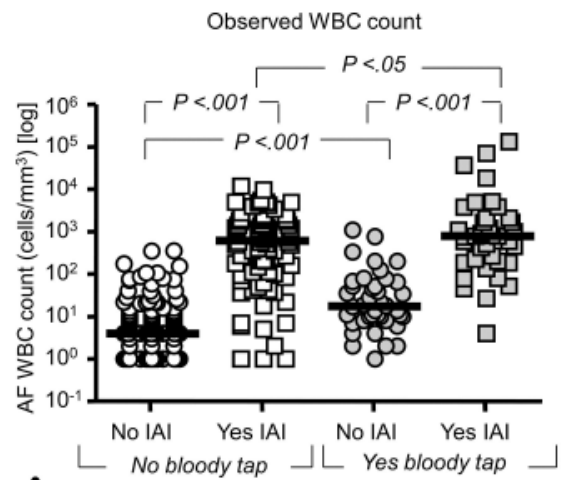

A

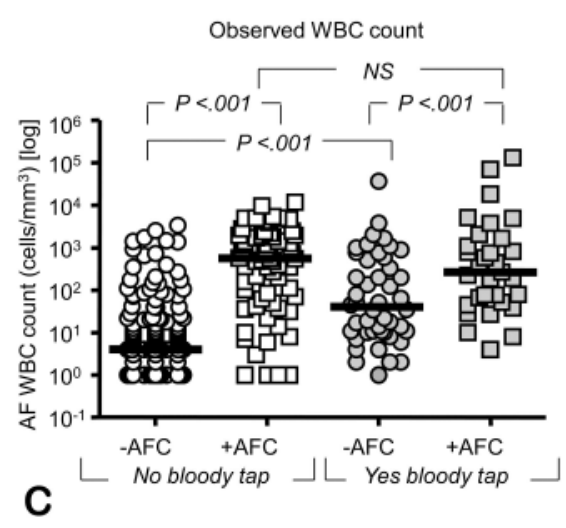

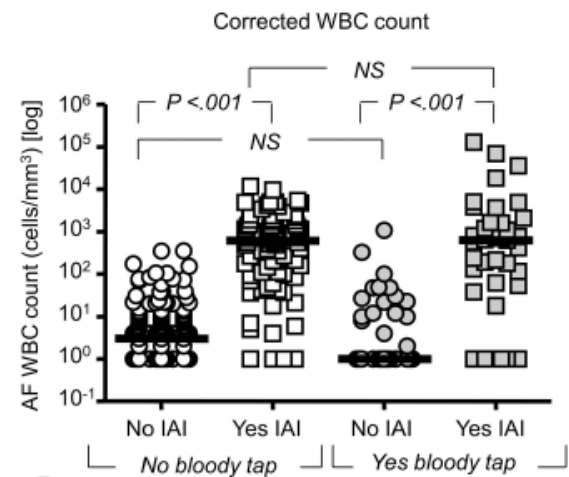

B

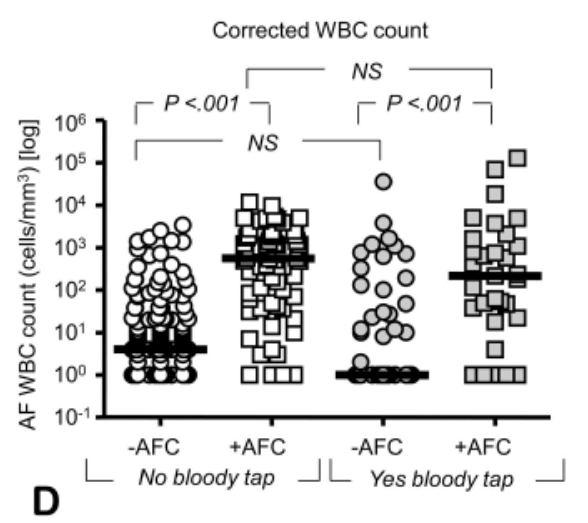

Fig. 2.

Distribution of the observed (A) and corrected (B) white blood cell (WBC) counts in patients grouped by presence of intraamniotic inflammation (IAI), "bloody tap" status of the amniotic fluid (AF), or both. Distribution of the observed (C) and corrected (D) WBC counts in patients grouped by results of AF culture (AFC), bloody tap status of the AF, or both. Data presented in logarithmic format. The thick black lines represent the median of the respective group of samples. 


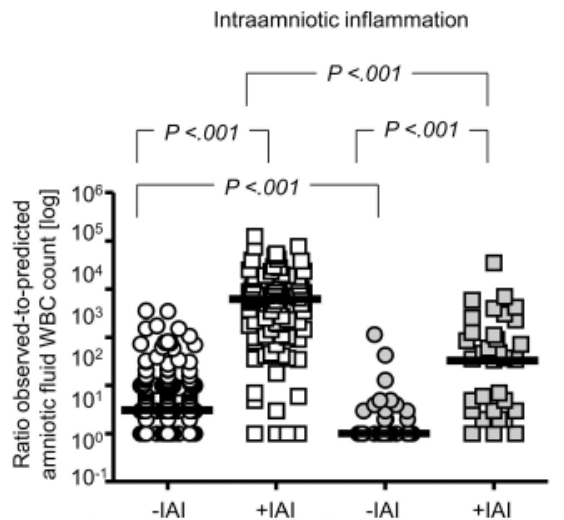

A

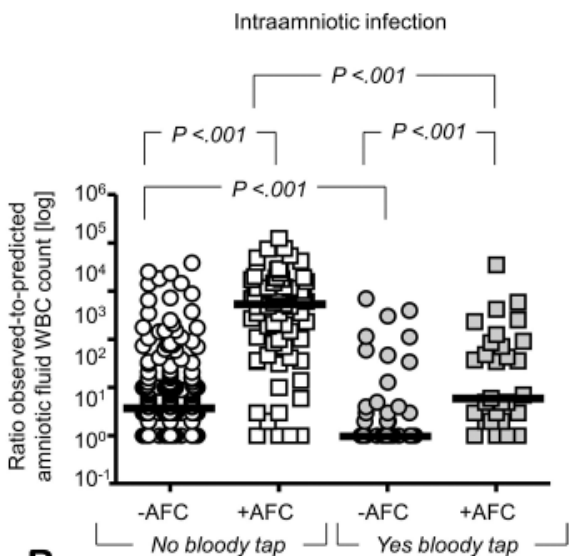

B

Fig. 3.

A. Distribution of the observed-to-predicted white blood cell (WBC) count in patients grouped by the results of the intraamniotic inflammation (IAI) and "bloody tap" status of the amniotic fluid (AF). B. Distribution of the observed-to-predicted WBC count in patients grouped by the results of the AF cultures (AFC) and bloody tap status of the AF. The thick black lines represent the median of the respective group of samples. 

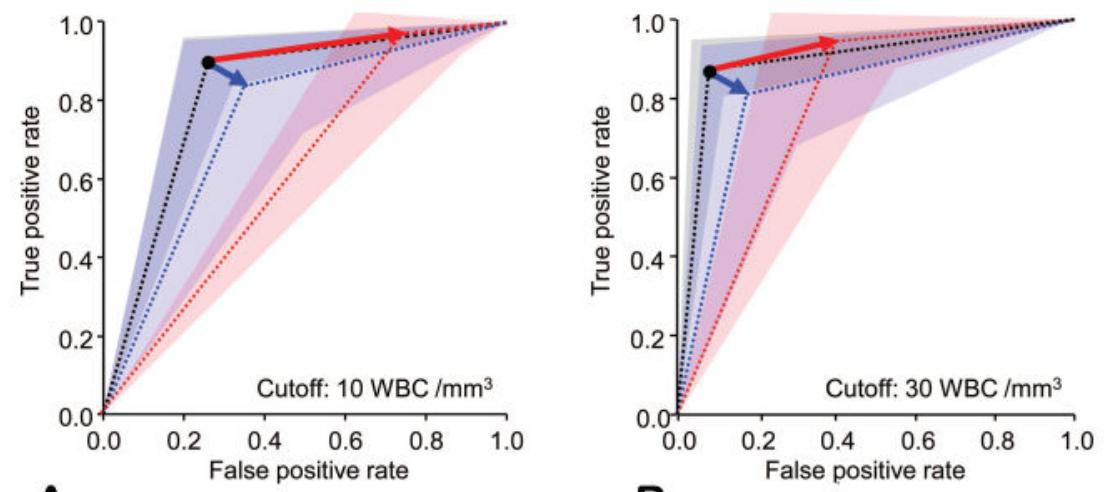

A

B
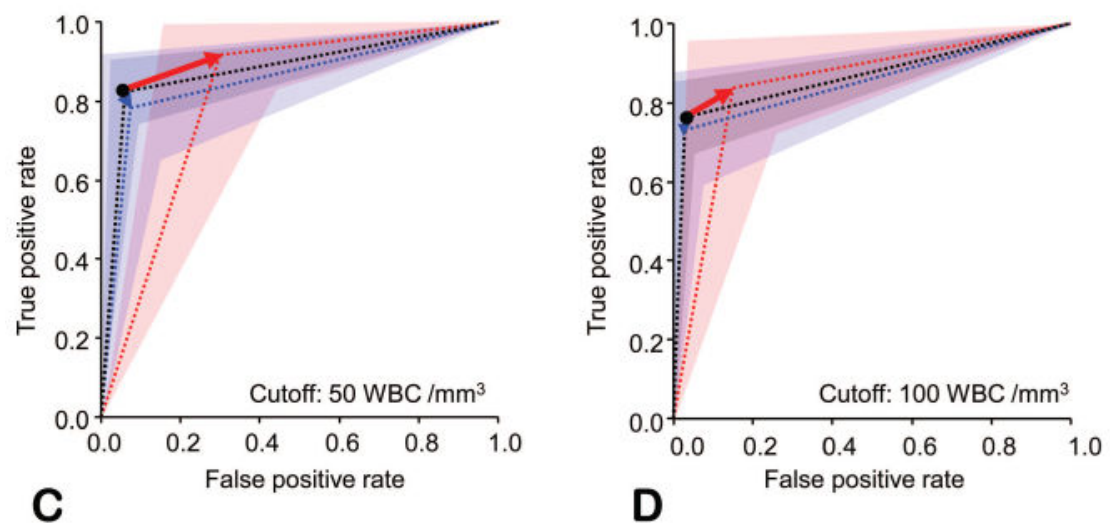

Fig. 4.

Likelihood ratio (LR) graphs and vectorial distance analysis displaying the clinical use of white blood cell (WBC) count correction in "bloody tap" amniotic fluid samples. The slopes of the positive LR (ascending line) and negative LR (horizontal line) along with their 95\% confidence intervals (shaded areas) for the three diagnostic tests are shown in black (observed WBC count non-bloody tap), red (observed WBC count bloody tap), and blue (corrected WBC count bloody tap), respectively. The red vector is illustrative of the level of bias imposed by a bloody tap procedure. The blue vector is a graphic representation of the proximity in diagnostic performance of the corrected WBC count compared with the best possible clinical scenario (non-bloody sample). The comparative analysis of the LRs was conducted using the same WBC cutoff for diagnosing intraamniotic inflammation: A. 10, B. 30, C. 50, and D. $100 \mathrm{WBC} / \mathrm{mm}^{3}$. 


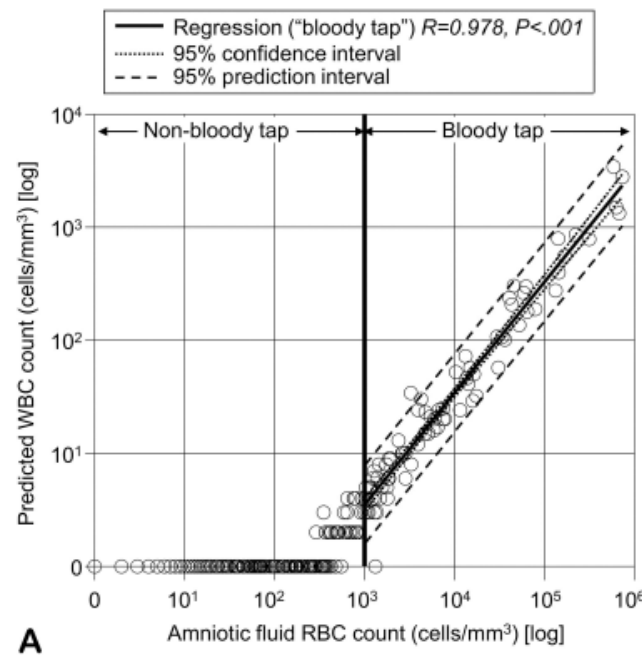

\begin{tabular}{|c|c|}
\hline $\begin{array}{c}\text { Amniotic fluid } \\
\text { RBC count } \\
\left(\text { cells/mm }{ }^{3}\right)\end{array}$ & $\begin{array}{c}\text { Approximate WBCs } \\
\text { needed to subtract } \\
\text { (cells/mm }{ }^{3} \text { ) }\end{array}$ \\
\hline Less than 1,000 & 0 \\
$1,000-2,999$ & 5 \\
$3,000-9,999$ & 20 \\
$10,000-29,999$ & 50 \\
$30,000-59,999$ & 170 \\
$60,000-99,999$ & 190 \\
$100,000-299,999$ & 560 \\
Greater than 300,000 & 1,500 \\
\hline
\end{tabular}

Fig. 5.

A. Log-log plot of the relationship between the levels of blood contamination of amniotic fluid (AF) reflected by the red blood cell (RBC) count and predicted white blood cell (WBC) count. B. Number of WBC count (median value) that should be subtracted from the amniotic fluid WBC count to determine rapidly the level of true leukocytosis for different levels of blood contamination of AF samples. 
Table 1

Observed and Corrected White Blood Cell Counts Based on Inflammatory Status of the Amniotic Fluid, Results of Microbial Cultures, and Amniocentesis Specimen Classification as a "Bloody Tap"

\begin{tabular}{|c|c|c|c|c|}
\hline Group & $\begin{array}{l}\text { Bloody Tap (More Than 1,000 } \\
\text { RBCs/mm } / \mathrm{mm}^{3} \text { ) }\end{array}$ & $\begin{array}{c}\text { Observed WBC Count } \\
\left(\text { cells } / \mathbf{m m}^{3}\right)\end{array}$ & $\begin{array}{l}\text { Corrected WBC Count } \\
\left(\text { cells } / \mathbf{m m}^{3}\right)\end{array}$ & $P$ \\
\hline \multicolumn{5}{|c|}{$\begin{array}{l}\text { Intraamniotic inflammation (MR score } \\
3-4)\end{array}$} \\
\hline \multirow[t]{2}{*}{ No $(\mathrm{n}=240)$} & No $(\mathrm{n}=200)$ & $4(1-10)$ & $3(1-10)$ & .330 \\
\hline & Yes $(n=40)$ & $18(9-58)$ & $0(0-17)$ & $<.001$ \\
\hline \multirow[t]{2}{*}{ Yes $(n=117)$} & No $(\mathrm{n}=80)$ & $616(105-1,730)$ & $615(105-1,729)$ & .848 \\
\hline & Yes $(n=37)$ & $778(197-2,062)$ & $629(61-1,765)$ & $<.001$ \\
\hline \multicolumn{5}{|c|}{ Intraamniotic infection (positive AFC) } \\
\hline \multirow[t]{2}{*}{ No $(n=260)$} & No $(\mathrm{n}=214)$ & $4(1-11)$ & $4(1-11)$ & .340 \\
\hline & Yes $(n=46)$ & $40(11-511)$ & $0(0-101)$ & $<.001$ \\
\hline \multirow[t]{2}{*}{ Yes $(n=97)$} & No $(n=66)$ & $561(62-1,790)$ & $560(61-1,790)$ & .814 \\
\hline & Yes $(\mathrm{n}=31)$ & $267(58-1,528)$ & $215(26-1,487)$ & $<.001$ \\
\hline
\end{tabular}

RBC, red blood cell; WBC, white blood cell; MR, Mass Restricted; AFC, amniotic fluid culture.

Data are median (interquartile range), unless otherwise specified, and analyzed by two-way repeated measures analysis of variance after logarithmic transformation of data. 


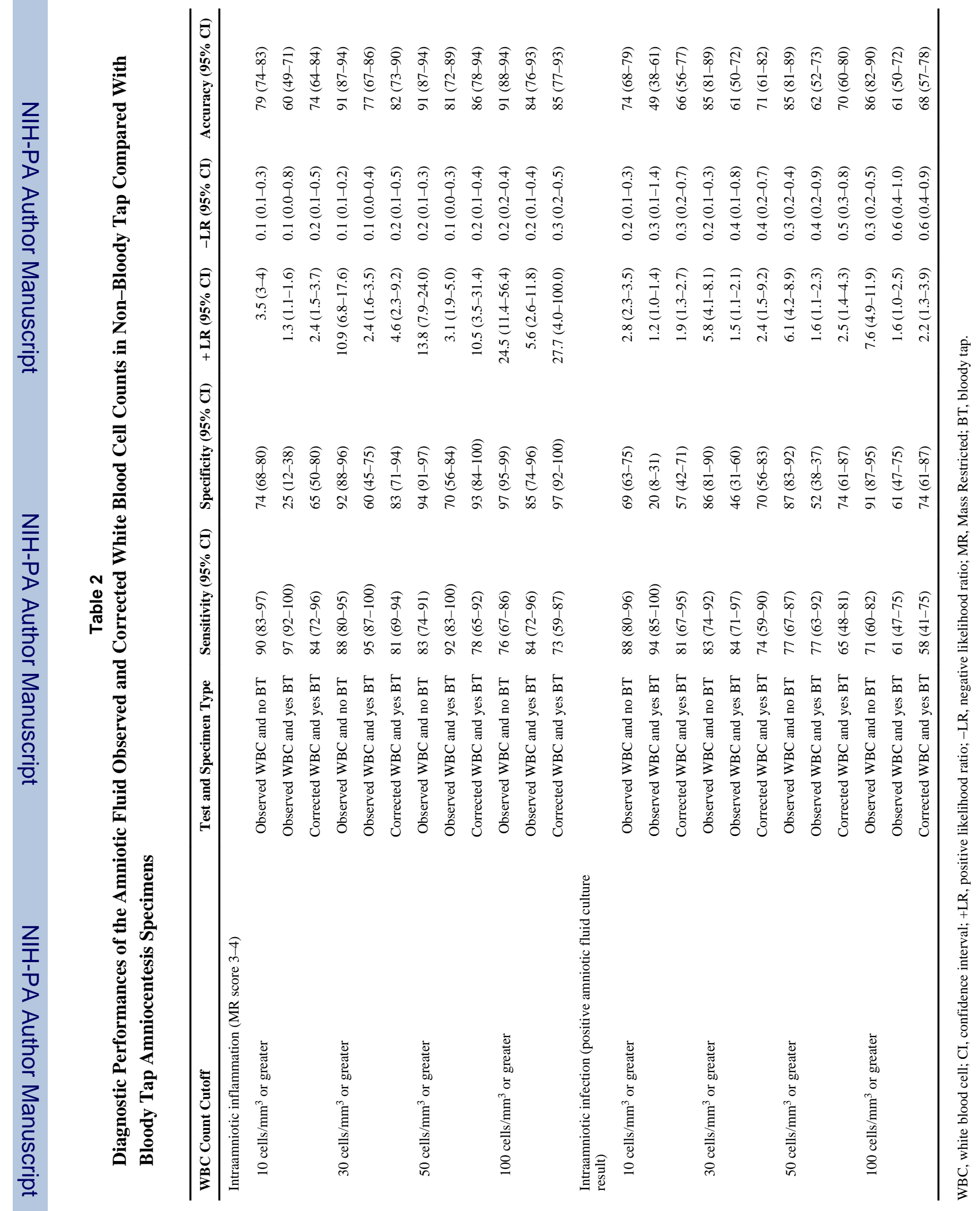

